\title{
L1 transfer, definiteness and specificity of determiners in L2 English
}

Sviatlana Karpava

University of Central Lancashire, Cyprus

https://doi.org/10.36505/ExLing-2016/07/0014/000273

\begin{abstract}
This study investigates L1 transfer from Cypriot Greek (CG), definiteness and specificity of determiners in L2 English. 100 CG undergraduate students (ages 17-23) participated in the study. The linguistic (socio-economic) background questionnaires were used. Their written corpus (100 essays) was analysed in terms of determiner production. They were also offered an elicitation task based on Ionin et al. (2003, 2004), which was focused on elicitation of definite determiner the in [+def; +spec] and [+def; - spec] environments and indefinite determiner $a$ in $[-$ def; + spec] and [-def; - spec] environments. The results of the study showed that the most problematic condition for CG students was [-def; +spec] with target indefinite determiner as they fluctuated in their written production between target and non-target settings.

Key words: determiners, definiteness, specificity, L1 transfer
\end{abstract}

\section{Introduction}

It was found that L2 English acquisition of articles is a very difficult process (Huebner, 1983; Master, 1987; Parrish, 1987; Robertson, 2000; Leung, 2001; Ionin et al., 2008). L2 leaners make omission or substitution errors (Larsen-Freeman, 1975; Thomas, 1989; Parodi et al, 1997; Hawkins et al., 2006). L2 learners either have access to Universal Grammar (UG), directly or via their L1, which is in line with the domainspecific view of L2 acquisition, or they use general learning mechanisms such as statistical learning, which is in line with the domain-general view (Ionin et al., 2008).

Definite articles are presuppositional expressions, while indefinite articles are quantificational expressions, as for the latter there is no prior presupposition or mentioning (Heim, 1991). In English, definite article the presupposes that the referent has been established by prior knowledge or discourse and this knowledge is shared by both a listener and a speaker (Ionin, 2003, 2006). Learning of articles involves formmeaning mapping. Definiteness is one of the cross-linguistic semantic universals, the other is specificity. L2 learners have access to both universals and they fluctuate between them. Ionin et al. (2003, 2004, 2008) observed that L2 learners of English have more accurate performance on $[+\mathrm{def} ;+$ spec] and [-def; -spec], when there is agreement

ExLing 2016: Proceedings of 7 th Tutorial and Research Workshop on Experimental Linguistics, 27 June - 2 July 2016, Saint Petersburg, Russia 
between definiteness and specificity, than on [+def; -spec] and [-def; + spec], when the two universals are in conflict. English articles encode definiteness rather than specificity, therefore L2 English input provides target-like definiteness patterns and L2 learners with a higher level of proficiency might be more successful than those with a lower one.

L1 Cypriot Greek (CG) has articles, which means that L2 learners of English with CG background would either transfer semantics of Greek article into English or fluctuate between definiteness and specificity semantic universals provided by UG (Ionin et al., 2003, 2004, 2008).

The aim of this study is to examine L2 acquisition of English definiteness and specificity of determiners, whether L1 transfer overrides fluctuation or fluctuation overrides L1 transfer and whether amount and quality of L2 input, level of proficiency and age affect L2 learners' production with respect to definite and indefinite articles.

\section{Study}

100 CG undergraduate students (ages 17-23, L2 proficiency: beginners, intermediate and advanced) participated in the study. The linguistic (socio-economic) background questionnaires were used. Their written corpus (100 essays) was analysed in terms of determiner production. They were also offered an elicitation task based on Ionin et al. (2003, 2004), which was focused on elicitation of definite determiner the in $[+$ def; + spec $]$ and $[+$ def; -spec] environments and indefinite determiner $a$ in [-def; +spec] and [-def; -spec] environments. The participants were offered to choose from three options each time (the, $a$ or $\varnothing$ ), there were 10 items for each condition. The task also investigated whether L2 learners of English transfer from L1 and they were asked to choose the appropriate variant (the, $a$ or $\varnothing$ ) in such semantic and syntactic environments, where CG and English differ in terms of article use (Holton et al., 2004; Buschfeld, 2013). There were also distractor items focused on the use of various tenses.

\section{Results and discussion}

The results of the study showed that the most problematic condition for CG students was [-def; +spec] with target indefinite determiner as they fluctuated in their written production between target $(42.55 \%)$ and non-target $(57.45 \%)$ settings. They mainly substituted indefinite article $a$ by the $(52.12 \%)$ or used null determiner $(5.31 \%)$. As far as other conditions concerned, for [+def; +spec] condition they had $76.38 \%$ target the and $23.62 \%$ non-target $(12.55 \%$ indefinite article or $11.07 \%$ omission); for [ + def; - spec] condition they used target the $(73.40 \%)$ and $26.60 \%$ non-target $(20.21 \%$ indefinite article and $6.39 \%$ 
omission); and for [-def; -spec] condition they had target $a(78.29 \%)$ and $21.71 \%$ non-target (12.34\% definite article and 9.37\% null article), see Table 1.

Table 1. Definite vs. indefinite article production in four environments.

\begin{tabular}{|l|l|l|l|l|}
\hline \hline Environment & target the & non-target & non-target $a$ & non-target $\varnothing$ \\
\hline$[+$ def; + spec $]$ & $76.38 \%$ & $23.62 \%$ & $12.55 \%$ & $11.07 \%$ \\
\hline [+def; -spec] & $73.40 \%$ & $26.60 \%$ & $20.21 \%$ & $6.39 \%$ \\
\hline Environment & target $a$ & non-target & non-target the & non-target $\varnothing$ \\
\hline [-def; + spec $]$ & $42.55 \%$ & $57.45 \%$ & $52.12 \%$ & $5.31 \%$ \\
\hline [-def; -spec] & $78.29 \%$ & $21.71 \%$ & $12.34 \%$ & $9.37 \%$ \\
\hline \hline
\end{tabular}

According to one-way $A N O V A$, age seems to be an important factor for the production of target the determiner in [+def; +spec] environment/condition: Sig 2-tailed .005. Age of onset to L2 English seems to be important for the target production of definite determiner the in [+def; -spec] condition Sig 2-tailed.047.

According to paired samples t-test, there is a statistically significance between the target production of indefinite determiner $a$ in [-def; + spec] and [-def; -spec] conditions: $t(99)=11.861, p=.000$; between the target production of definite determiner the in $[+\mathrm{def} ;+\mathrm{spec}]$ condition and target production of indefinite article $a$ in [-def; +spec] condition: $t(99)=8.702, p=.000$; between target production of definite article the in [+def; -spec] condition and target production of indefinite article $a$ in [def; +spec] condition: $t(99)=6.290, p=.000$.

It was found that L2 learners of English transfer from L1 CG, but the rate of transfer is low: they used definite determiners with proper names and places $(24.69 \%$ ), before time expressions (17.66\%), with nouns that are additionally modified by a demonstrative and possessive $(12.77 \%)$, quantifiers all and the whole (36.18\%), with most of (54.47\%). They tend to omit indefinite articles in predicate DPs after verbs to be and to become (21.28\%), with expression like (21.71\%), in direct object position with the verb have $(32.77 \%)$, see Table 2 .

According to one-way $A N O V A$, age is an important factor for the omission of articles in time expressions due to L1 transfer: Sig 2-tailed .005. The results of the study showed that fluctuation overrides L1 only for [-def; +spec] condition, when two semantic universals, definiteness and specificity are not in agreement. This finding is in line with Ionin et al. (2008) and Trenkic (2000) as L2 learner had an overall better performance in the use of definite than indefinite articles. Age is a statistically important factor for definite/indefinite article acquisition in L2 English, but not the level of proficiency, quantity and quality of input. 
CG participants transfer from L1 and might not pay attention to discourse-based triggers in L2 English.

Table 2. L1 transfer from CG.

\begin{tabular}{|c|c|c|c|}
\hline \multirow{2}{*}{\multicolumn{4}{|c|}{\begin{tabular}{|l|l|l|} 
target $\varnothing$ non-target & non-target $\varnothing$ \\
with nroper names/nlace names
\end{tabular}}} \\
\hline & & & \\
\hline \begin{tabular}{|l|l|}
$75.31 \%$ & $24.69 \%$ \\
\end{tabular} & $12.12 \%$ & $7.68 \%$ & $4.89 \%$ \\
\hline
\end{tabular}

preceding time destination, hours, weekdays, months, years and before seasons

\begin{tabular}{|l|l|l|l|l|}
\hline $82.34 \%$ & $17.66 \%$ & & $10.63 \%$ & $7.03 \%$ \\
\hline
\end{tabular}

with nouns which are additionally modified by a demonstrative and possessive \begin{tabular}{|l|l|l|l|l}
\hline $87.23 \%$ & $12.77 \%$ & $4.89 \%$ & $7.88 \%$
\end{tabular}

with nouns that are additionally modified by the quantifiers all and whole

\begin{tabular}{|l|l|l|l|}
\hline $63.82 \%$ & $36.18 \%$ & $21.70 \%$ & $14.48 \%$
\end{tabular}

in predicate DPs after the verbs to be and to become, predicate structures, simple DPs

\begin{tabular}{|c|c|c|c|}
\hline \begin{tabular}{|l|l|}
$78.72 \%$ & $21.28 \%$ \\
\end{tabular} & $16.18 \%$ & $5.10 \%$ & \\
\hline \multicolumn{4}{|l|}{ with like } \\
\hline \begin{tabular}{|l|l|}
$78.29 \%$ & $21.71 \%$ \\
\end{tabular} & $15.33 \%$ & $6.38 \%$ & \\
\hline \multicolumn{4}{|l|}{ with most of } \\
\hline \begin{tabular}{|l|l|}
$45.53 \%$ & $54.47 \%$ \\
\end{tabular} & & $49.36 \%$ & $5.11 \%$ \\
\hline \multicolumn{4}{|c|}{ in direct object position with the verb have } \\
\hline \begin{tabular}{|l|l|}
$67.23 \%$ & $32.77 \%$ \\
\end{tabular} & $30.63 \%$ & $2.14 \%$ & \\
\hline
\end{tabular}

\section{References}

Buschfeld, S. 2013. English in Cyprus or Cyprus English? An Empirical Investigation of Variety Status. Amsterdam: John Benjamins.

Heim, I. 1991. Artikel und Definitheit. In Stechow, A and Wunderlich, D. (eds.), Semantik: Ein internationales Handbuch der zeitgenossischen Forschung, 487-535. Berlin: de Gruyter.

Ionin, T. 2003. Article Semantics in Second Language Acquisition. Unpublished doctoral dissertation, MIT.

Ionin, T. 2006. This is definitely specific: specificity and definiteness in article systems. Natural Language Semantics 14, 175-234.

Ionin, T., Ko, H. and Wexler, K. 2004. Article semantics in L2-acquisition: the role of specificity. Language Acquisition 12, 3-69.

Ionin, T., Zubizarreta, M.L. and Maldonado, S.B. 2008. Sources of linguistic knowledge in the second language acquisition of English articles. Lingua 118, 554-576.

Trenkic, D. 2000. The acquisition of English articles by Serbian speakers. Unpublished $\mathrm{PhD}$ dissertation. University of Cambridge. 